\title{
Are Trials of Intravascular Infusions of Autologous Mesenchymal Stem Cells in Patients with Multiple System Atrophy Currently Justified, and Are They Effective?
}

$\mathrm{M}$ ultiple system atrophy (MSA) is a rare, sporadic, fatal multisystem progressive disorder characterized by autonomic failure, orthostatic hypotension, neurogenic bladder/erectile dysfunction, cerebellar ataxia, corticospinal dysfunction, and parkinsonism that may be poorly L-dopa responsive. ${ }^{1}$ MSA in Asia has a predominance of cerebellar ataxia (MSA-C), whereas the United States and Europe have a predominance of parkinsonism (MSA-P). Mean survival from diagnosis to death is 7 to 10 years. $^{2,3}$ Currently, there are no treatments that halt or reverse disease progression. Neuropathologically, MSA is characterized by glial cytoplasmic inclusions of abnormally aggregated $\alpha$-synuclein with loss of nearby neurons. ${ }^{4}$ Disease progression is thought to be causally linked to accumulation and aggregation of $\alpha$-synuclein, and accordingly considerable effort has gone into devising strategies to combat these events. ${ }^{5}$ Recent work with transgenic models suggests that a redistribution of neuronal to oligodendroglial $\alpha$-synuclein may take place in the pathophysiology of MSA, but the precise mechanism by which aggregation leads to neuronal loss is unproven. ${ }^{6}$ Recent evidence suggests a resulting deficiency of growth factors, especially brain-derived neurotrophic factor and glial cell-derived neurotrophic factor, which might respond to infusion of mesenchymal stem cells (MSCs). ${ }^{7}$

Against this background, the open-label study by Lee et $\mathrm{al}^{8}$ reporting that MSC infusion halts the progression of MSA sparked interest in the potential value of MSCs in the treatment of MSA. A commentary on the publication criticized the limited scientific basis for the study and the lack of blinding. ${ }^{9}$ The current publication ${ }^{10}$ has greater scientific credibility. The study was performed at a single center and comprises a doubleblind, placebo-controlled treatment trial on 33 subjects with MSA-C (17 placebo and 16 MSCs). MSCs or placebo was infused intra-arterially sequentially into the internal carotid arteries and dominant vertebral artery followed by subsequent infusions (at 1, 2, and 3 months) intravenously. The primary endpoint consisted of a mixed model approach to the total unified MSA rating score (UMSARS). This scoring system rates the symptoms and functional status of relevant activities of daily living (UMSARS I) and a motor examination (UMSARS II). Secondary endpoints included UMSARS II, cerebral glucose metabolism, and estimated gray matter density. The results included improvement in both UMSARS II and UMSARS total, with the secondary endpoints all trending in the appropriate direction. This potentially important study stimulates a number of questions. Does the study demonstrate that we have a treatment for MSA? Is the treatment safe? What is the mechanism of action? How do the MSCs breach the blood-brain barrier (BBB)?

One key issue in MSC treatment of MSA is developing access to target tissue across the BBB. This is a significant barrier to the delivery of stem cells, although it appears to be less intact in MSA than in normal subjects. In a study that evaluated the integrity of the BBB with 2 clinical methods, the barrier was less tight in MSA than in normal subjects, ${ }^{11}$ and the impairment correlated with the severity of disease (UMSARS score). Nevertheless, the remaining barrier continues to constitute a major hurdle to MSC access. Because growth factors also do not generally cross the BBB, peripheral stem cell delivery may not reach target neurons in the relevant areas of the brain. Intravenous infusion results in poor delivery to target tissue because of entrapment and loss of MSCs, especially in lungs, but also in liver and spleen, ${ }^{12,13}$ resulting in minimal engraftment of stem cells in the brain. In contrast, intra-arterial administration of stem cells significantly improves engraftment into target brain tissue. ${ }^{14}$ The safety of intra-arterial injection is controversial, and both development of microstrokes and increased mortality have been reported. ${ }^{15}$

The study by Lee et al is clearly a potentially important step forward in the treatment of a fatal disease. 
The investigators met their primary endpoint coupled with some positive secondary endpoints, including improvement in ${ }^{18} \mathrm{~F}$-fluorodeoxyglucose positron emission tomography glucose metabolism, cortical density, and cognitive scores. Despite the positive findings, this study leaves unresolved a number of issues on efficacy. First, this is a single-center study, whereas a study involving multiple centers might have provided more robust data. Second, the study was performed on a relatively small number of subjects. Third, the study was confined to MSA-C, whereas the majority of MSA subjects in the USA and Europe have MSA-P. Fourth, authors found significant improvement in total UMSARS and UMSARS II (motor examination), but not in the quality of life scale (UMSARS I), meaning that over a year, the level of functioning was insufficient to improve the patients' quality of life. Fifth, the results over time suggest only a transient improvement, with the treatment losing much of its benefit within months after the treatment.

There are significant concerns regarding the safety of intra-arterial infusions. A significant percentage $(29 \%$ of MSC-treated and 35\% of placebo-treated) patients developed ischemic lesions on magnetic resonance imaging, including 1 subject who had transient neurologic deficits. The authors seem rather cavalier in dismissing this problem as simply due to the intra-arterial approach. The mechanism of benefit is also uncertain. If MSCs provide growth or other factors that delay entry of neurons into the apoptotic pathway, and the benefit is transient, then repeated infusions are likely necessary. The approach carries an unacceptable risk. Whither the path forward? Clearly this current approach is not a cure for MSA, but may have the potential of delaying progression. In comparison to the previous unblinded study of MSCs, the study design has improved with blinding, but the scientific approach of the study is only modestly more robust. What needs to be done? Should further studies with MSCs be done on patients with MSA? This study is tantalizing but clearly not definitive. Consequently, first the study should be repeated in a multicenter double-blind study at some point in time. Second, such a study should be done only when a safer method of MSC delivery is available. Third, the study should be carried out with both MSA-P and MSA-C subjects. Fourth, as the benefits appear to be transient, the issue of mode of administration is important, and future approaches will need to couple safer access with a treatment paradigm that ensures sustained improvement.

\section{Potential Conflicts of Interest}

S.G.: grants/grants pending, NIH, NINDS; travel expenses, NIH; paid review activities, Pfizer, Janssen AI, Elan.
Phillip A. Low, MD ${ }^{1}$

Sid Gilman, MD 2

${ }^{1}$ Department of Neurology

Mayo Clinic

Rochester, MN

${ }^{2}$ Department of Neurology

University of Michigan

Ann Arbor, MI

\section{References}

1. Gilman S, Wenning GK, Low PA, et al. Second consensus conference on the diagnosis of multiple system atrophy. Neurology 2008;71:670-676.

2. Geser F, Wenning GK, Seppi K, et al. Progression of multiple system atrophy (MSA): a prospective natural history study by the European MSA Study Group (EMSA SG). Mov Disord 2006;21: 179-186.

3. Lipp A, Sandroni P, Ahlskog JE, et al. Prospective differentiation of multiple system atrophy from Parkinson's disease, with and without autonomic failure. Arch Neurol 2009;66:742-750.

4. Wakabayashi K, Takahashi H. Cellular pathology in multiple system atrophy. Neuropathology 2006;26:338-345.

5. Windisch M, Wolf HJ, Hutter-Paier B, Wronski R. Is alpha-synuclein pathology a target for treatment of neurodegenerative disorders? Curr Alzheimer Res 2007;4:556-561.

6. Rockenstein E, Ubhi K, Inglis C, et al. Neuronal to oligodendroglial alpha-synuclein redistribution in a double transgenic model of multiple system atrophy. Neuroreport 2012;23:259-264.

7. Ubhi K, Rockenstein E, Mante M, et al. Neurodegeneration in a transgenic mouse model of multiple system atrophy is associated with altered expression of oligodendroglial-derived neurotrophic factors. J Neurosci 2010;30:6236-6246.

8. Lee PH, Kim JW, Bang OY, et al. Autologous mesenchymal stem cell therapy delays the progression of neurological deficits in patients with multiple system atrophy. Clin Pharmacol Ther 2008; 83:723-730.

9. Quinn N, Barker RA, Wenning GK. Are trials of intravascular infusions of autologous mesenchymal stem cells in patients with multiple system atrophy currently justified, and are they effective? Clin Pharmacol Ther 2008;83:663-665.

10. Lee $\mathrm{PH}$, Lee JE, Kim HS, et al. A randomized trial of mesenchyma stem cells in multiple system atrophy. Ann Neurol 2012;72:32-40.

11. Song SK, Lee SK, Lee JJ, et al. Blood-brain barrier impairment is functionally correlated with clinical severity in patients of multiple system atrophy. Neurobiol Aging 2011;32:2183-2189.

12. Fischer UM, Harting MT, Jimenez F, et al. Pulmonary passage is a major obstacle for intravenous stem cell delivery: the pulmonary first-pass effect. Stem Cells Dev 2009;18:683-692.

13. Kraitchman DL, Tatsumi M, Gilson WD, et al. Dynamic imaging of allogeneic mesenchymal stem cells trafficking to myocardial infarction. Circulation 2005;112:1451-1461.

14. Guzman R, De Los Angeles A, Cheshier S, et al. Intracarotid injection of fluorescence activated cell-sorted CD49d-positive neural stem cells improves targeted cell delivery and behavior after stroke in a mouse stroke model. Stroke 2008;39:1300-1306.

15. Walczak P, Zhang J, Gilad AA, et al. Dual-modality monitoring of targeted intraarterial delivery of mesenchymal stem cells after transient ischemia. Stroke 2008;39:1569-1574.

DOI: 10.1002/ana.23655 